\title{
physiotherapie
}

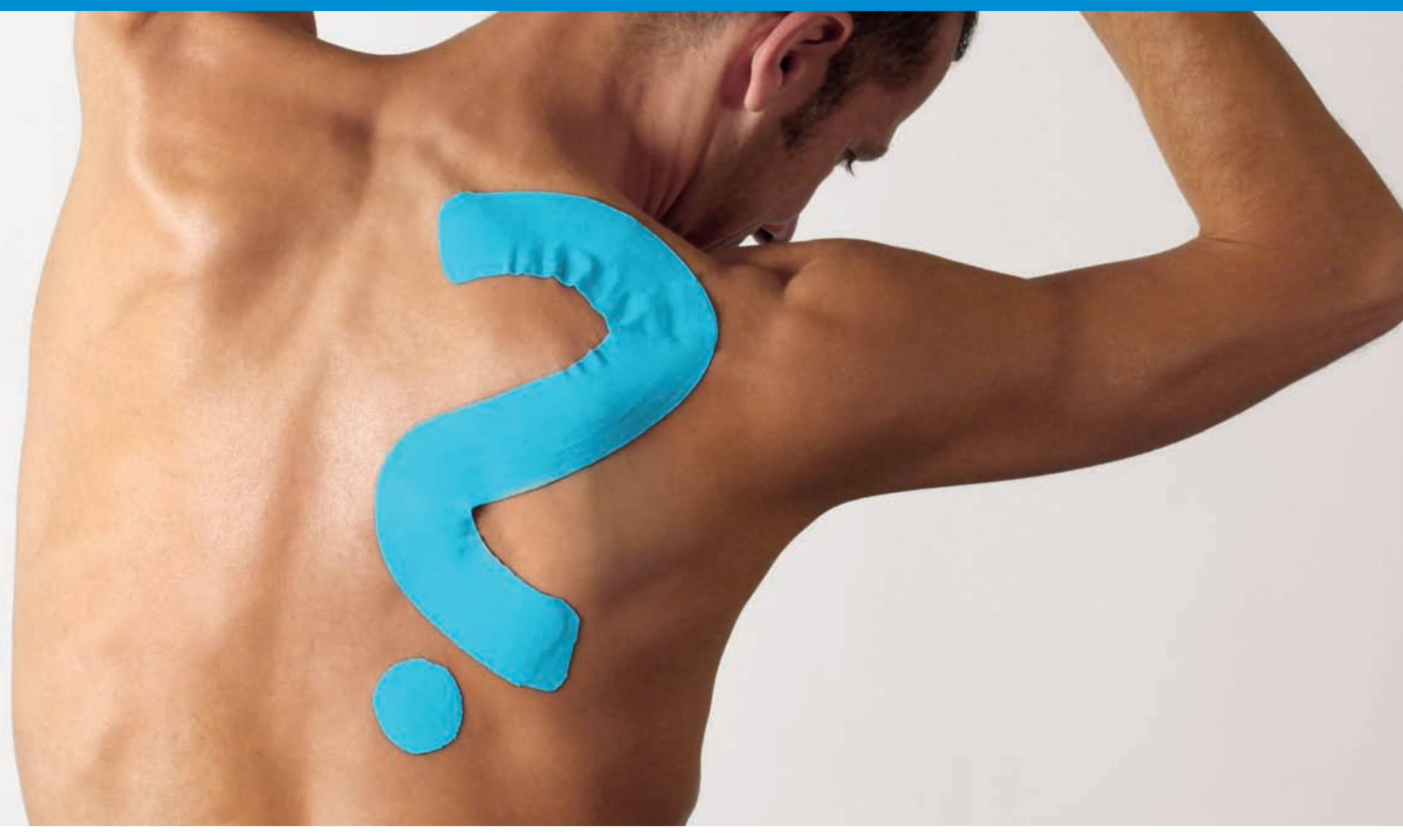

\section{El Fenómeno}

\section{SOFTTAPING-KURSE IM ÜBERBLICK Kaum eine Olympiade, Sportschau oder Amateur-}

sportveranstaltung findet heutzutage ohne bunt getapte Sportler statt. Softtaping ist im Trend, die Zahl der Fortbildungsanbieter wächst stetig. Die Unterschiede sind auf den ersten Blick kaum erkennbar. physiopraxis stellt elf Anbieter vor.

nter einem Phänomen verstand schon Immanuel Kant ein Ding oder ein Ereignis, das in seiner Erscheinung oder Wirkung keine Beziehung zur eigentlichen Daseinsform hat. So ähnlich könnte man auch den derzeitigen Boom um das Softtape beschreiben: Jeder kennt es, jeder liebt es. Ob Spitzensportler, Hobbyläufer oder Couchpotato, fast jeder hat solch einen bunten Streifen schon getragen oder zumindest gesehen. Schmerzlinderung, propriozeptive Unterstützung und Förderung des Lymphflusses sind Auszüge aus der breiten Wirkungspalette, die die zahlreichen SofttapeHersteller und -Kursanbieter anpreisen.

Wer sich animiert fühlt, eine Softtape-Fortbildung zu besuchen, steht allerdings - bei der großen Vielfalt der Kursangebote - vor der schwierigen Aufgabe, die für ihn richtige aufzuspüren. Die in den 1970er Jahren von Dr. Kenzo Kase entwickelte ursprüngliche Kinesio-Tape-Methode kann man in Deutschland nicht erlernen. Stattdessen gibt es hierzulande eine Vielzahl von Fortbildungsanbietern, die die Methode mehr oder minder direkt von Kase gelernt haben. Ein hart umkämpfter Markt, in dem sich nicht alle Anbieter grün sind.

Auf den ersten Blick scheinen die Kurse ähnlich zu sein. Wer sich jedoch mit den Details beschäftigt, wird mit Sicherheit ein „buntes Wunder“ erleben. physiopraxis hat bei elf Fortbildungsanbietern nachgefragt. 
Medical Taping Concept

Grundkurs: 295€, 2 Tage

Aufbaukurs: $295 €, 2$ Tage

Lymphtaping: 220€, 1 1/2 Tage
Nachdem wir 1998 die Kinesio-Tape-

Methode in Europa eingeführt haben, entstanden neue Methoden wie „Medical Taping“, „Lymph Taping“ und „Meridian Taping“. Daraufhin haben wir, die PhysioTape International Academy, all diese Methoden im Medical Taping Concept zusammengefasst. Die ursprüngliche Kinesio-TapeMethode von Dr. Kenzo Kase veränderten wir nur gering. Allerdings war sie bei weitem noch nicht ausgereizt. So behandeln wir beispielsweise nicht nur Muskeln, sondern auch das Lymphsystem.

Worin unterscheidet sich Ihre Methode von den anderen?
Wir haben jahrelange Erfahrung, da wir die ersten Anbieter auf dem Markt waren. Wir haben eine neue Tape-Variante kreiert das CureTape „Punch“ mit patentiertem Lochmuster. Wir legen viel Wert auf einen hohen Qualitätsstandard, und unsere Seminare haben ein hohes fachliches Niveau.
Medical Taping Concept der Schulungsgruppe SG (süddeutsche Sektion)

Basiskurs MTC: 325€, 3 Tage

Aufbaumodule (Schulter, Fuß/Knie, Sportverletzungen und Lymph-Tape): je $150 €$, je 1 Tag Aufbaumodule des Neurophysiologischen Taping Concepts (NTC)/Kinetic Tape/ Kinetic Taping NTC 1 (untere Körperhälfte) und NTC 2 (obere Körperhälfte): je $280 €$, je 2 Tage

2000 führten wir das elastische Taping in Süddeutschland mit dem ersten Kurs ein. Die ursprüngliche Kinesio-Tape-Methode ist wenig praxisorientiert und oft ineffektiv. Dr. Kenzo Kase hatte im Gegensatz zu uns kein Interesse an Anatomie, Biomechanik und komplexen Zusammenhängen. Wir arbeiten neuromuskuloskeletal und nicht kinesiologisch oder esoterisch.

Unser Basis-Kurs grenzt sich von den anderen ab, da er sehr funktionell ist und sich an Anatomie, Biomechanik, aktuellem Stand der Wissenschaft, Physik und Chemie orientiert. Wir arbeiten in befundorientierten und dysfunktionsspezifischen Ausgangsstellungen und mit spezifischen Applikationen wie Slider-Techniken bei neuralen Problematiken. Die Anlage überprüfen wir mit spezifischen Tests und runden sie mit speziellen Übungen ab.

Farben spielen für uns nur bedingt eine Rolle. Die Tape-Farbe hängt beispielsweise davon ab, ob man auffällig tapen kann. Für uns ist Farbe subjektiv, abhängig vom Lichteinfluss und der Lichtbrechung sowie ein SäureBasen-Indikator. Farbe bedeutet Chemie und mehr Festigkeit, da sich die Farbpartikel unterschiedlich stark und dicht an die Baumwollfäden anordnen.

Tape-Name: KIRA Sports Tape Hersteller: KTC-Germany GmbH Das KIRA Sports Tape, das wir selbst entwickelt haben, ist geschützt und steht nur den von uns ausgebildeten Therapeuten zur Verfügung.
Das CureTape, das wir nach wissenschaft lichen Untersuchungen entwickelt haben, bietet eine TÜV-geprüfte Qualität.
Ja, wir kleben die Cross-Patches, wie wir sie nennen, direkt auf energiegeladene Stellen wie Trigger-, Schmerzund Akupunkturpunkte.
Ja.
Arbeiten Sie mit Cross-Tapes? 


\section{physiotherapie}

\section{Kinematic Taping}

Kursdauer und -kosten
Basismodul KT0: 280€, 2 Tage

Aufbaumodul KT1: $280 €$, 2 Tage

Aufbaumodul KT2: 280€, 2 Tage

Neurodynamics: $560 €, 4$ Tage

Handrehabilitation: $140 €, 1$ Tag

Fitness/Wellness: 280€, 2 Tage
Dolo-Taping

1. Seminarteil (Grundlagen des Dolo-Tapings): $295 €, 2$ Tage

2. Seminarteil (Aufbauseminar/Lymph-Taping): $295 €, 2$ Tage

Dolo-Taping für Therapeuten mit Kenntnissen in anderen flexiblen Taping-Therapien: $135 €, 1$ Tag
Wie ist Ihre Methode entstanden und wie hebt sie sich vom ursprünglichen Kinesio-Tape ab?

Worin unterscheidet sich lhre Methode von den anderen?

Spielen die Farben bei Ihrer Methode eine Rolle?
Wir haben die ursprüngliche Kinesio-TapeMethode von Dr. Kenzo Kase überarbeitet und in das Gesamtmanagement nach IFOMPT'-Standard integriert. Unser Konzept zeichnet sich aus durch Clinical Reasoning $\left(\mathrm{OMT}^{2}\right)$ sowie befund- und behandlungsorientierte Tape-Anlagen spezifisch für Dysfunktionen und Bewegungsstörungen. Dr. Kenzo Kase wollte vor allem die Eigenschaften der Haut nachahmen. Die spezifische Beeinflussung von dynamischem, mechanorezeptivem Input hat er im Gegensatz zu uns nicht berücksichtigt.

Unser Anspruch: mehr Wissen, mehr Können, mehr Erfolg. Unsere Dozenten sind erfahrene Kliniker und Experten in Sportphysiotherapie und internationaler Manueller Therapie. Sie sind akademisch, pädagogisch und wissenschaftlich ausgebildet. Unsere Anlagen heben sich durch den Stretch, die Ausgangsstellung, die Kombination von Tape-Anlagen, die Anlagenlänge und die passenden Übungen sicht- und spürbar von anderen ab. Zudem setzen wir die Tape-Elastizität zwischen 0 und $100 \%$ konsequent ein.

Die Farben spielen nur insofern eine Rolle, als dass sie der Beeinflussung des frequenzempfindlichen autonomen Nervensystems dienen. In Bezug auf die Farben fehlt jegliche Evidenz.
Unsere Erfahrungen mit der ursprünglichen Kinesio-Tape-Methode von Dr. Kenzo Kase waren unbefriedigend, da die Heilungsrate zu niedrig war. Das Dolo-Taping hat im Gegensatz zur ursprünglichen Kinesio-Tape-Methode eine ganzheitliche Betrachtungsweise. Wir forschen nach den Ursachen und beseitigen diese.
Bei Patienten mit Epicondylitis beheben wir beispielsweise zuerst Beinlängenunterschiede, Becken- und WS-Fehlstellungen. Narben prüfen wir auf Störfelder, tapen und entstören sie mit dem entsprechenden Tape (Farbe). Dann tapen wir alle triggerpunktschmerzhaften Muskeln im Schulter-Nacken-, WS-, Oberarm-, Unterarm-, Brust- und Beckenbereich. So reduzieren wir nicht nur die Schmerzen, sondern erreichen meist eine Heilung. Andere Methoden würden nur den Ellenbogen tapen.

Farben haben einen Anteil von etwa $70 \%$ der Gesamtwirkung. Unsere Tapes sind in den Volltönen Blau, Gelb und Rot sowie in den Mischtönen Orange und Grün. Schwarz und Pink gibt es bei uns nicht, da Pink aus 21 Einzelfarben besteht und Schwarz für Tod steht. Verwendet man eine Farbe falsch, können sich die Beschwerden deutlich verschlimmern.

Tape-Name: Dolo-Tape Hersteller: Mcksure

Wir haben das Dolo-Tape 2007 selbst entwickelt. Es hebt sich in Stärke, Elastizität, Kleber und Farbmischung von den bislang erhältlichen Tapes ab. 
Die ursprüngliche Kinesio-Tape-Methode von Dr. Kenzo Kase beschränkt sich vor allem auf die Therapie muskuloskeletaler Erkrankungen. Basierend auf den Erfahrungen von Dr. Dieter Sielmann und seinen Dialogen mit Ärzten und Therapeuten wurde und wird das Medi-Taping ständig weiterentwickelt und ausgeweitet.

So wird es nun auch in der Neurologie, Chirurgie, Pädiatrie, Geriatrie und Gynäkologie angewendet.

Wir stellen Fehlstatiken vor allem im Bereich des lliosakralgelenkes und die damit einhergehende Fehlbelastung des Bewegungsapparates in den Mittelpunkt der Therapie, da fast alle Schmerzpatienten eine funktionelle Beinlängendifferenz aufweisen.
Neben der neuromuskulären und myofaszialen Kinesio-Tape-Methode von Dr. Kenzo Kase wurde ein reflexorientiertes Konzept entwickelt. Hierfür hat Reinhold Roth (Physio- und Manualtherapeut) Elemente der Manuellen Therapie, Osteopathie, Brügger, Sporttherapie und der Traditionellen Chinesischen Medizin mit seinen Erfahrungen zu einem ganzheitlichen Konzept verknüpft. Das Senso-Taping, das unter anderem Muskeln, Faszien, Gelenkkapseln, Nerven und Organe stimuliert, spielt darin eine wichtige Rolle.

Im Vordergrund steht bei uns die Behandlung von Schmerzsyndromen (akut und chronisch) sowie die Therapie funktioneller, durch Fehlhaltungen verursachter Beschwerden. Wir integrieren in das Senso-Taping-Konzept verschiedene physiotherapeutische Techniken und berücksichtigen energetische und psychoenergetische Aspekte der Traditionellen Chinesischen Medizin.
Als wir vor vier Jahren begonnen haben, eine Kinesio-Tape-Fortbildung zu entwickeln, wollten wir keine neue Methode kreieren. Ziel war eine praxisnahe Technik, die sich an der ursprünglichen KinesioTape-Methode von Dr. Kenzo Kase orientiert. Wir schauten uns die bisherigen TapeMethoden an, um zu entscheiden, welche Ansätze für uns interessant sind. Uns fiel auf, dass einige Anbieter die ursprüngliche Methode stark modifiziert hatten. Andere hatten zu wenig Praxisnähe. Wir konzipierten eine Methode, die diese Lücke füllt.

Wir bieten ein sehr gutes Preis-LeistungsVerhältnis. Manche Anbieter sind qualitativ hochwertig, aber sehr teuer. Andere sind günstig, da sie aber stark von der ursprünglichen Methode abweichen und oft keine klare Linie erkennbar ist, ist ihr Konzept von geringerem Nutzen. Unser Konzept ist nach Krankheitsbildern geordnet und daher sehr übersichtlich, leicht anwendbar und kompakt.
Die Wahl der Farbe hat einen nicht zu unterschätzenden Anteil an der Behandlung. Wir wählen die Farben unterstützend zur jeweiligen Indikation und Therapie aus. Wir verwenden die fünf klassischen Farben Beige, Grün, Gelb, Blau und Rot.
Wir verwenden ausschließlich blaue und rote Tapes. Die Farben spielen dabei eine wesentliche Rolle, da sie im Sinne der Traditionellen Chinesischen Medizin verschiedene Reaktionen und Assoziationen auslösen. Die Farbe beeinflusst die Stimmung und energetisch auch das Meridiansystem.
Da wir uns stark an der ursprünglichen Kinesio-Tape-Methode orientieren, spielen Farben für uns eine wichtige Rolle: Rot als Farbe des Feuers (tonisierend), Blau als Farbe des Wassers (detonisierend) und Hautfarben beispielsweise für Anlagen im Gesicht.

\section{Tape-Name: Medi-Tape}

Hersteller: 3M

Wir haben das Medi-Tape selbst entwickelt. Es unterliegt ständigen Qualitätskontrollen.
Tape-Name: Sensotape

Hersteller: Die Fachwelt Verlags- und

Handelsgesellschaft mbH

Sensotape zeichnet sich durch eine lang anhaltende Klebewirkung aus, bei einer gleichzeitig besonders guten Hautverträglichkeit (hypoallergen, atmungsaktiv, geruchsneutral).

Ja, wir nutzen die Gitter-Tapes, wie wir sie nennen, als Verbindung zwischen und auf Meridianen sowie bei Narben, Triggerund Ashi-Punkten.
In unseren Kursen verwenden wir Schupp Tapes Kinesiologie. Die Teilnehmer entscheiden frei, woher sie ihr Material beziehen. Wir haben keine Exklusivverträge mit TapeHerstellern. Unsere Kernkompetenz ist die Fortbildung, nicht der Tape-Vertrieb.

Ja, wir arbeiten mit Cross-Tapes, vor allem im Bereich der Triggerpunkttherapie oder auf kleinen Gelenken wie dem Kiefergelenk. 


\section{physiotherapie}

K-Taping

Kursdauer und -kosten

Wie ist Ihre Methode entstanden und wie hebt sie sich vom ursprünglichen Kinesio-Tape ab?

Worin unterscheidet sich Ihre Methode von den anderen?

\begin{abstract}
K-Taping Pro: $550 €, 3$ Tage
K-Taping Gyn: 165€, 1 Tag

K-Taping Care Plus: 275€, 1 1⁄2 Tage

K-Taping Lymph: 165€, 1 Tag

K-Taping Sport: $360 €, 2$ Tage

K-Taping Osteopathie: $375 €$, 2 Tage

K-Taping Neuro: $375 €, 2$ Tage

K-Taping Golf: $375 €, 2$ Tage

K-Taping Refresher: $340 €, 2$ Tage
\end{abstract}

Birgit Kumbrink gründete 1998 das KinesioTaping-Schulungszentrum und legte damit den Grundstein für die Kinesio-TapingAusbildung in Deutschland und Europa. Aufgrund der vielen neuen Anlagekonzepte, basierend auf Studien, gründete Birgit Kumbrink 2006/2007 die Internationale K-Taping Academy. Wir haben die internationalen Schulungskonzepte durch Anlagen für den Bereich Pädiatrie, Neurologie, Gynäkologie und Ergotherapie erweitert und in 40 Ländern weltweit mit Unterstützung vieler Verbände durchgeführt.

Wir haben ein internationales Ausbildungskonzept, das von Verbänden in vielen Ländern anerkannt wird. Wir führen Studien mit namhaften Universitätskliniken durch. Wir bieten ein internationales Therapeutenforum. In unseren Schulungen zeigen wir nicht nur Anlagen, sondern auch die Kombinationen des K-Tapings mit Therapieansätzen wie Manuelle Medizin und Bobath.

\section{K-Active Taping}

Grundkurs: 278€, 1 11/2 Tage

Sondertechniken kompakt (Aufbaukurs):

$278 €, 1 \frac{1}{2}$ Tage

Refresher: $168 €, 1 \mathrm{Tag}$

Sonderkurse: Sport: $168 €, 1$ Tag

Wirbelsäule/ISG: $98 €, 1 / 2 \mathrm{Tag}$

Lymphologie: $98 €, 1 / 2 \mathrm{Tag}$

Gynäkologie/Geburtshilfe: $98 €, 1 / 2$ Tag

PreCut Tageskurs: $178 €$

K-Active ist aus der Firma Kinesio entstanden. Unsere Senior-Instruktoren wurden durch Dr. Kenzo Kase in Japan und in Europa ausgebildet. Es besteht bis zum heutigen Tag zur Weiterentwicklung und Optimierung noch reger Austausch mit Japan. Wir haben die ursprüngliche Kinesio-Tape-Methode durch Forschung unter anderem auf dem Gebiet der myofaszialen Systeme zu einem ganzheitlichen Konzept gemacht.

Alle Kinesio-Tape-Methoden basieren auf der japanischen Form. Viele Anbieter ließen sich aber im Gegensatz zu uns nicht das Verständnis dieser ursprünglichen Methode in Japan oder durch japanische Instruktoren vermitteln. Die Publikationen von Dr. Kenzo Kase wurden oft nur minimal verändert, um es als neue Methode präsentieren zu können. Wir bieten einen individuellen, ganzheitlichen Ansatz, den es unseres Wissens nach so nur bei uns gibt.
Spielen die Farben bei Ihrer Methode eine Rolle?
Wir verwenden die vier Originalfarben Cyan, Magenta, Beige und Schwarz. Die farblich unterschiedlichen Tapes haben jedoch exakt die gleichen Eigenschaften. Wir wählen die Farben in Anlehnung an die Farblehre unterstützend zur Therapie aus. Unsere Methode wirkt durch die richtige Anlagetechnik, nicht durch die Farbe.
Jede Farbe hat eine Schwingung, die unterschiedliche Reaktionen im Körper auslöst. Daher testen wir mit einem „abgespeckten“ kinesiologischen Muskeltest, welche Farbe das System am meisten stärkt. So hält die Anlage länger, es kommt zu weniger Hautreaktionen, und auch der psychologische Effekt für den Patienten ist besser.

Tapename: K-Active Tape Hersteller: Nitto Denko Corporation, Japan Nitto Denko (die einzige in Japan ansässige Firma, die vom deutschen TÜV ISO-zertifiziert ist) hat mit Dr. Kenzo Kase das erste kinesiologische Tape entwickelt. Wir bieten es in Europa als Einzige an.

Ja, wir vertreiben auch Cross-Tapes und zeigen in unseren Kursen die Anwendungsmöglichkeiten. Sie sind jedoch kein Behandlungsschwerpunkt.
Ja, das Cross-Tape und dessen Anwendung hat ebenfalls die K-Taping Academy in Deutschland und Europa eingeführt und verbreitet.

\section{Arbeiten Sie mit Cross-Tapes?}

Tapename: K-Tape
Hersteller: biviax

der Firmeiten ausschließlich mit dem Kals Standardtape bei sämtlichen Forschungen und Studien eingesetzt wird. 
Basiskurs: $270 €, 2$ Tage

Aufbaukurs: $270 €, 2$ Tage

Sport Performance/Trauma: $150 €, 1$ Tag

Neurologie: $140 €, 1 \mathrm{Tag}$

Myofasziale Meridiane: $300 €, 2$ Tage
Dynamic-Tape-Praxis-Seminar:

$50 €, 2$ 1⁄2 Stunden (über die Firma Sissl)
Myofasziales Kinesiology Taping ist ein neues ganzheitliches myofasziales Konzept, das einen komplett anderen Ursprung und einen ganz anderen Ansatz hat als die anderen kinesiologischen Taping-Arten. Wir fokussieren uns auf Faszien. Es basiert auf dem Anatomy Trains Konzept und auf Wiederherstellung der myofaszialen Balance durch Aktivierung und Entspannung der Myofaszien. Es wurde 2006 von Markus Erhard entwickelt.
Unser Tape basiert nicht auf der KinesioTape-Methode von Dr. Kenzo Kase. Ryan Kendrick (Physiotherapeut vieler Sportler, unter anderem von Tennisspieler Greg Rusedski) hat zuerst mit kinesiologischen Tapes experimentiert. Für seine Sportler benötigte er aber ein flexibleres Tape. $\mathrm{Er}$ entwickelte das Dynamic Tape, das wir vor einem Jahr als erstes biomechanisches Tape auf den Markt brachten. Das kinesiologische Tapen wollen wir nicht ersetzen, sondern ergänzen. Unser Tape hilft bei vielen Beschwerden, aber nicht bei allen.

Unser Tape ist extrem dehnbar - in Längsund Querrichtung, so können wir komplette Muskelketten tapen. In unseren Kursen geben wir nicht vor, wie man einzelne Muskeln oder Krankheiten tapt. Wir erklären lediglich die Charakteristik unseres Tapes und zeigen exemplarisch ein paar Anlagen. Biomechanisches Wissen und eine gute Anamnese reichen anschließend aus, um individuell tapen zu können. Wir versuchen mit unserem Tape unter anderem die Biomechanik zu korrigieren.

Wir verwenden hautfarbige Tapes, die wahlweise mit oder ohne Tattoo-Muster verziert sind. Beide Tapes sind jedoch gleich wirkungsvoll. Das Tattoo-Muster ist lediglich ein Marketing-Gag, damit sich unser Tape von den kinesiologischen Tapes unterscheidet.
Unser Tape wirkt rein mechanisch und neurologisch, daher spielen die Farben bei uns keine Rolle und richten sich beispielsweise nach der Trikotfarbe eines Sportlers. Wir verwenden schwarze, beige, gelbe, rote, grüne, weiße, hell- und dunkelblaue, pink- und orangefarbene Tapes.
Tapename: FLEXOTAPE
Hersteller: Flexotape
Das FLEXOTAPE ist ein einzigartiges und innovatives, flexibles Tape, das sich auf $140 \%$ der originalen Länge dehnen lässt. Durch die besonders hohe Klebekraft wird es sehr oft

\author{
Tapename: Dynamic Tape \\ Hersteller: PosturePals Pty Ltd \\ Unser Tape ist CE-zertifiziert, doch es \\ kann - wie bei allen anderen Tape- \\ Materialien auch - in seltenen Fällen zu \\ Unverträglichkeiten kommen.
} im Spitzensport weltweit eingesetzt. 